TRANSACTIONS OF THE

AMERICAN MATHEMATICAL SOCIETY

Volume 352, Number 3, Pages 1277-1298

S 0002-9947(99)02442-3

Article electronically published on October 15, 1999

\title{
NATURAL EXTENSIONS FOR THE ROSEN FRACTIONS
}

\author{
ROBERT M. BURTON, CORNELIS KRAAIKAMP, AND THOMAS A. SCHMIDT
}

\begin{abstract}
The Rosen fractions form an infinite family which generalizes the nearest-integer continued fractions. We find planar natural extensions for the associated interval maps. This allows us to easily prove that the interval maps are weak Bernoulli, as well as to unify and generalize results of Diophantine approximation from the literature.
\end{abstract}

\section{INTRODUCTION}

Although D. Rosen [Ro] introduced his infinite family of continued fractions in the mid-1950s, it is only very recently that there has been any investigation of its metric properties [S], [N2], [GH]. Here we find explicit planar natural extensions for each of the interval maps associated to the Rosen fractions. The systems we find are similar to those found for certain semi-regular continued fraction maps in [NIT].

The explicit natural extensions allow us to strengthen metric results in the literature - in particular, we give invariant measures for all Rosen maps. We show that the maps are each weak Bernoulli (and thus ergodic) with respect to the invariant measures. We also extend techniques of [K1] and [K2] to unify and extend various arithmetic and Diophantine results. The Rosen fractions are a generalization of the nearest integer continued fractions $[\mathrm{R}],[\mathrm{K} 1]$; our results restrict to include this classical case.

It is a pleasant surprise that although the basic shape of the region for the system of the natural extension changes quite drastically with the basic index of the fractions, the Radon-Nikodým derivative with respect to two-dimensional Lebesgue measure remains constant. The limit, in a natural sense, of these Rosen maps is the even partial quotients map, see say [KL]. Only in this limiting case does the planar region admit a point at which the Radon-Nikodým derivative is infinite. Thus, one sees quite clearly the cause of the transition from finite to infinite invariant measure in the limit of this family of maps.

Proving the weak Bernoulli property in this setting is complicated by the fact that the natural partitions for the maps are such that there are cylinder sets which are not mapped onto the whole interval. We circumvent this difficulty by finding an induced system which does not have this defect and then showing that the weak Bernoulli property can be spread back to the whole system.

Received by the editors August 1, 1997.

2000 Mathematics Subject Classification. Primary 11J70, 37A25.

The first author was partially supported by AFOSR grant 93-1-0275 and NSF grant DMS 96-26575. The third author was partially supported by the Nederlandse Organisatie voor Wetenschappelijk Onderzoek (NWO). 
The generic quality of Diophantine approximation of the Rosen transformations also limits to that of the even partial quotients map, despite the fact that the Rosen maps are naturally divided into two subfamilies - those of odd index and those of even. The fine behavior of the transformations is starkly different in these settings.

We mention some related theory. Underlying the family of transformations which we discuss here is an example of what J. Wolfart [Wo] called a "discrete deformation of Fuchsian groups". Wolfart showed that for any Riemann surface with a cusp, there is a family of Riemann surfaces with quotient singularities which has the given cusped surface as its limit (in the Chabauty topology). In our setting, the limit surface is the arithmetic surface which corresponds to the so-called theta-group. It might be very interesting to give the analog of our results for a general discrete deformation and family of associated interval maps.

RMB and TAS thank the SSOR group of TU Delft for its friendly hospitality while this work was being completed. CK would like to thank the Department of Mathematics of Oregon State University for its friendly hospitality during several visits.

\section{HECKE GROUPS AND CONTINUED FRACTIONS}

The groups which underlie our interval maps are Fuchsian groups of the first kind - discrete groups of $P S L(2, \mathbf{R})$, acting upon the Poincaré upper half-plane by Möbius (fractional linear) transformations, with all of $\mathbf{R}$ as their limit sets. We avoid most of this theory, but the reader may wish to consult [B] for a related discussion.

Let $\lambda=\lambda_{q}$ equal $2 \cos \pi / q$ for $q \in\{3,4, \ldots\} ; S=\left(\begin{array}{cc}1 & \lambda \\ 0 & 1\end{array}\right)$ and $T=\left(\begin{array}{rr}0 & -1 \\ 1 & 0\end{array}\right)$. Then the group $G_{q}$ generated by $S$ and $T$ is called the Hecke (triangle) group of index $q$. All relations in the group arise from $T^{2}=I$ and $U^{q}=I$, where $U=S T$ and $I$ represents the projective identity.

D. Rosen [Ro] defined a continued fraction related to $G_{q}, q \geq 4$. Fix some such $q$ and let $I_{q}=[-\lambda / 2, \lambda / 2)$. Then the map

$$
\begin{aligned}
f_{q}: & I_{q} \rightarrow I_{q}, \\
& x \rightarrow \frac{\varepsilon}{x}-\left\lfloor\frac{\varepsilon}{x \lambda}+\frac{1}{2}\right\rfloor \lambda,
\end{aligned}
$$

where $\varepsilon$ is the sign of $x$, gives a shift map on continued fraction expansions of the type

$$
\begin{aligned}
x & =\frac{\varepsilon_{1}}{r_{1} \lambda+f_{q}(x)} \\
& =\frac{\varepsilon_{1}}{r_{1} \lambda+\frac{\varepsilon_{2}}{r_{2} \lambda+\ddots}} \\
& =:\left[\varepsilon_{1}: r_{1}, \varepsilon_{2}: r_{2}, \ldots\right],
\end{aligned}
$$

where $\varepsilon_{i} \in\{ \pm 1\}$ and $r_{i} \in \mathbf{N}$. We call this the Rosen or $\lambda$-expansion ( $\lambda \mathrm{CF}$ ) of $x$. Of course, the various $\varepsilon_{i}$ and $r_{i}$ depend on $x$, but we will only indicate that dependence when it seems necessary to stress it. In analogy to the classical setting, we call $x$ a $G_{q}$-irrational if $x$ has a Rosen expansion of infinite length. There are restrictions on the set of admissible sequences of $\varepsilon_{i}$ and $r_{i}$. These are given explicitly in [Ro]. For 
$G_{q}$-irrationals, these restrictions are determined simply by the orbit of $\lambda / 2$. This is visible in the natural extensions which we construct; see Figures 1 and 2 in $\S 3$.

The metric theory requires a discussion of the cylinder sets for the transformation $f_{q}$. We let $\Delta\left[\varepsilon_{1}: r_{1}, \ldots, \varepsilon_{n}: r_{n}\right]$ be the set of $x \in I_{q}$ such that the expansion of $x$ begins as $\left[\varepsilon_{1}: r_{1}, \ldots, \varepsilon_{n}: r_{n}\right]$. One defines $\Delta\left[\varepsilon_{1}: r_{1}, \ldots, \varepsilon_{n}:\right]$ similarly.

Many standard formulas for continued fractions hold for the Rosen fractions; for proofs of the following, one can directly generalize results in [K2], §1. Letting

$$
p_{n} / q_{n}=\left[\varepsilon_{1}: r_{1}, \varepsilon_{2}: r_{2}, \ldots, \varepsilon_{n}: r_{n}\right],
$$

we find that

$$
\begin{aligned}
& p_{n}=r_{n} \lambda p_{n-1}+\varepsilon_{n} p_{n-2} \\
& q_{n}=r_{n} \lambda q_{n-1}+\varepsilon_{n} q_{n-2} .
\end{aligned}
$$

If we replace the "tail" of a continued fraction, we have the following formula (with a small abuse of notation):

$$
\left[\varepsilon_{1}: r_{1}, \varepsilon_{2}: r_{2}, \ldots, \varepsilon_{n}:\left(r_{n} \lambda+y\right)\right]=\frac{p_{n}+p_{n-1} y}{q_{n}+q_{n-1} y} .
$$

To obtain precise values of specific expansions, we utilize a sequence of polynomials in $\lambda$, already discussed by H. Weber [W], see in particular his $\S 144$. (The identitites we use are elementary, but we find it convenient to refer to Weber's results.)

$$
\left\{\begin{array}{c}
B_{0}=0 ; \quad B_{1}=1 \\
B_{n}=\lambda B_{n-1}-B_{n-2}, \quad n \geq 2 .
\end{array}\right.
$$

Note that

$$
U^{n}=\left(\begin{array}{ll}
B_{n+1} & -B_{n} \\
B_{n} & -B_{n-1}
\end{array}\right)
$$

In what follows, we use the identities implied by these being of determinant 1 without further comment.

Now, $[\mathrm{W}], \S 144$, equation 15 , gives

$$
B_{n}=\frac{\sin n \pi / q}{\sin \pi / q}
$$

Interpreting $U$ as a Möbius transformation, by inspection one finds $U^{-1}(1 / x)=$ $1 / U(x)$ and thus, $U^{q-j}(1 / x) \cdot U(x)=1$. Since $U(x)=\lambda-1 / x$, we have $U^{j}(\lambda)=$ $\lambda-U^{q-j-2}(\lambda)$. Now, if $q=2 p$ is even one solves to find that $U^{p-1}(\lambda)=\lambda / 2$; similarly, if $q=2 h+3$ is odd, one finds that $U^{h}(\lambda)=1$. In a slightly more complicated way, again with $q=2 h+3$, we get $U^{h} \cdot S^{2} T \cdot U^{h-1}(\lambda)=2 / \lambda$. The above is in accordance with the formulas of [Ro]; in particular, using exponents to indicate repeated entries,

$$
-\lambda / 2=\left\{\begin{array}{cl}
{\left[(-1: 1)^{p-1}\right],} & \text { if } q=2 p, \\
{\left[(-1: 1)^{h},-1: 2,(-1: 1)^{h}\right],} & \text { if } q=2 h+3 .
\end{array}\right.
$$

In our construction, the orbit of $-\lambda / 2$ under $f$ is of extreme importance. We define $\phi_{j}$ to be $f^{j}\left(\frac{-\lambda}{2}\right)$, with $\phi_{0}=-\frac{\lambda}{2}$. Thus for even $q, \phi_{j}=S^{-1} T\left(\phi_{j-1}\right)$, for $j \in\{1, \cdots, p-1\}$ and $\phi_{p-1}=0$. For $q=2 h+3$, we have $\phi_{j}=S^{-1} T\left(\phi_{j-1}\right)$, for 
$j \in\{0, \cdots, h-1\} \cup\{h+1, \cdots, 2 h\} ; \phi_{h+1}=S^{-2} T\left(\phi_{h}\right)$ and $\phi_{2 h+1}=0$. That is, $\phi_{j}$ has for its expansion the $j$-th shift of that of $\phi_{0}$.

\section{Construction of the natural extensions}

In this section we explicitly find a planar natural extension for each of the Rosen interval maps. We commence by seeking a transformation which on the first coordinate is simply the interval map while on the second coordinate is directly related to the "past" of the first coordinate. The normalized invariant measure for this planar natural extension is readily found; projection gives the invariant measure for the Rosen map.

Note that for all $q \geq 4$ if $n \geq 2$ then $f_{q}$ maps the cylinder sets $\left(\frac{2}{(2 n+1) \lambda_{q}}, \frac{2}{(2 n-1) \lambda_{q}}\right)$ and $\left(\frac{-2}{(2 n-1) \lambda_{q}}, \frac{-2}{(2 n+1) \lambda_{q}}\right)$ onto $I_{q}$. If $n=1$, then the corresponding cylinders are not mapped surjectively to $I_{q}$. The reader may wish to compare the graph of some $f_{q}(x)$ with the systems of Figures 1 and 2 .

3.1. Even indices. We fix an even $q=2 p$, let $\lambda=\lambda_{q}$, and let $f(x)$ be $f_{q}(x)$ and

$$
\mathcal{T}(x, y)=\left(f(x), \frac{1}{r \lambda+\varepsilon y}\right),
$$

where we have suppressed the dependence of $r=r_{1}$ and $\varepsilon=\varepsilon_{1}$ on $x$. We now construct a union of rectangles as domain $\Omega$ in $\mathbf{R}^{2}$ upon which $\mathcal{T}$ is bijective.

Let $I$ be the interval $I:=\left[-\frac{\lambda}{2}, \frac{\lambda}{2}\right)$. As above, let $\phi_{j}=f^{j}(-\lambda / 2)$. Now let $J_{j}=\left[\phi_{j-1}, \phi_{j}\right)$ for $j \in\{1, \cdots, p-1\}$, and $J_{p}=\left[0, \frac{\lambda}{2}\right)$. Furthermore, let $K_{j}=$ $\left[0, L_{j}\right], j \in\{1, \cdots, p-1\}$, and $K_{p}=[0, R]$; see Figure 1 .

Theorem 3.1. Let $q=2 p$, with $p \geq 2$ and $\Omega=\bigcup_{k=1}^{p} J_{k} \times K_{k}$. If the relations

$$
\left\{\begin{array}{ll}
\left(\mathcal{R}_{0}\right): & R=\lambda-L_{p-1}, \\
\left(\mathcal{R}_{1}\right): & L_{1}=1 /(\lambda+R), \\
\left(\mathcal{R}_{j}\right): & L_{j}=1 /\left(\lambda-L_{j-1}\right) \\
\left(\mathcal{R}_{p}\right): & R=1 /\left(\lambda-L_{p-1}\right)
\end{array} \quad \text { for } j \in\{2, \cdots, p-1\}\right.
$$

all hold, then $\mathcal{T}: \Omega \rightarrow \Omega$ is bijective off of a set of Lebesgue measure zero.

Proof. It is easily seen that $\mathcal{T}$ is injective. We thus concern ourselves with the surjectivity.
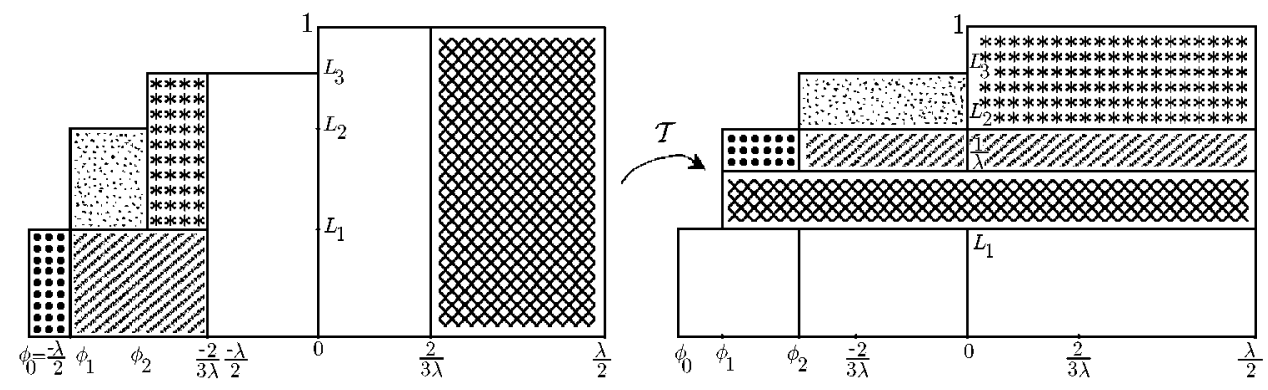

Figure $1 . \Omega$ and $\mathcal{T}(\Omega)$ for $q=8$. 
If $n \geq 2$, then

$$
\mathcal{T}(x, y)= \begin{cases}(f(x), 1 /(n \lambda+y)), \text { with } y \in[0, R] & \text { if } x \in\left(\frac{2}{(2 n+1) \lambda}, \frac{2}{(2 n-1) \lambda}\right), \\ (f(x), 1 /(n \lambda-y)), \text { with } y \in\left[0, L_{p-1}\right] & \text { if } x \in\left(\frac{-2}{(2 n-1) \lambda}, \frac{-2}{(2 n+1) \lambda}\right) .\end{cases}
$$

Thus,

$$
\begin{gathered}
\mathcal{T}\left(\left(\frac{-2}{(2 n-1) \lambda}, \frac{-2}{(2 n+1) \lambda}\right) \times\left[0, L_{p-1}\right]\right) \cup \mathcal{T}\left(\left(\frac{2}{(2 n+1) \lambda)}, \frac{2}{(2 n-1) \lambda)}\right) \times[0, R]\right) \\
=I \times\left[\frac{1}{n \lambda+R}, \frac{1}{n \lambda-L_{p-1}}\right] .
\end{gathered}
$$

But, the images corresponding to consecutive values of $n$ form layers, with "one above the next," because of condition $\mathcal{R}_{0}$. Now, by $\mathcal{R}_{1}$ and $\mathcal{R}_{p}$,

$$
L_{1}=1 /(\lambda+R)=1 /\left(2 \lambda-L_{p-1}\right),
$$

and we have that $I \times\left[0, L_{1}\right]$ is contained in the image of $\mathcal{T}$.

The image under $\mathcal{T}$ of $J_{p} \times K_{p}$ is $\left[\phi_{1}, \lambda / 2\right) \times[1 /(\lambda+R), 1 / \lambda]$. But, by $\mathcal{R}_{1}$, this image is $\bigcup_{j=1}^{p} J_{j} \times\left[L_{1}, 1 / \lambda\right]$.

We now address the region for which $f(x)=-1 / x-\lambda$. For $(x, y) \in\left[\phi_{0},-2 / 3 \lambda\right] \times$ $\left[0, L_{1}\right]$, one has $\mathcal{T}(x, y)=\left(S^{-1} T(x), 1 /(\lambda-y)\right)$. Thus, $\mathcal{R}_{2}$ shows that $\bigcup_{j=2}^{p} J_{j} \times$ $\left[1 / \lambda, L_{2}\right]$ is in the image of $\mathcal{T}$. Similarly, for $k \in\{1, \ldots, p-2\}, \mathcal{R}_{k+1}$ and $\mathcal{R}_{k+2}$ give that $\mathcal{T}$ takes $\left[\phi_{k},-2 / 3 \lambda\right] \times\left[L_{k}, L_{k+1}\right]$ onto $\bigcup_{j=k+1}^{p} J_{j} \times\left[L_{k+1}, L_{k+2}\right]$. Finally, $\mathcal{R}_{p-1}$ and $\mathcal{R}_{p}$ show that $\mathcal{T}$ takes $\left[\phi_{p-1},-2 / 3 \lambda\right] \times\left[L_{p-2}, L_{p-1}\right]$ onto $J_{p} \times\left[L_{p-1}, R\right]$.

Thus we have found $\Omega$ on which $\mathcal{T}$ is bijective, if we can assure that all $\mathcal{R}_{j}$ can be simultaneously satisfied.

Lemma 3.1. The system $\mathcal{R}_{0}, \mathcal{R}_{1}, \ldots, \mathcal{R}_{p}$ admits a unique real solution. For this solution, $R=1$.

Proof. Due to $\mathcal{R}_{0}$ and $\mathcal{R}_{p}$, any solution must have $R=1$. Now, the relations allow us to solve for the $L_{j}$.

It remains to show the relations are consistent. In fact, there is a cyclic relation on $R$. From $\mathcal{R}_{p}, \ldots, \mathcal{R}_{1}$ and then $\mathcal{R}_{0}$ one finds that

$$
R=\frac{1}{\lambda-\cdots \frac{1}{\lambda+R}}=\frac{1}{U^{p-1}(\lambda+R)}=\frac{1}{U^{p}(-1 / R)} .
$$

To prove our lemma, it suffices to show that this is consistent.

Since $U$ is of order $q, U^{p}$ is of order two. Hence $U^{p}$ has zero trace. That is, see (3), $B_{p+1}=B_{p-1}$, and one easily checks that $U^{p}(-1)$ equals 1 . That is, the value $R=1$ uniquely determines all of the $L_{j}$ without contradiction.

Now a Jacobian calculation for

$$
\mathcal{T}(x, y)=\left(\frac{\varepsilon}{x}-n \lambda, \frac{1}{n \lambda+\varepsilon y}\right)
$$

shows that $\mathcal{T}$ preserves the probability measure $\nu$ with density

$$
\frac{C}{(1+x y)^{2}}
$$


where $C$ is a normalizing constant, which we now determine.

Lemma 3.2. Let $q$ be even. The normalizing constant $C$ such that $\nu$ is a probability measure on $\Omega$ is given by

$$
C=1 / \ln \left[\left(1+\cos \frac{\pi}{q}\right) / \sin \frac{\pi}{q}\right]
$$

Proof. Integration gives

where

$$
C^{-1}=\ln \left[V \cdot\left(1+\frac{\lambda}{2}\right)\right]
$$

$$
V:=\prod_{j=1}^{p-1} \frac{1+\phi_{j} \cdot L_{j}}{1+\phi_{j-1} \cdot L_{j}}
$$

To evaluate $V$, we note that $\phi_{p-1}=0$ and $\phi_{p-2}=-1 / \lambda$; indeed,

$$
\phi_{p-1-j}=-\frac{B_{j}}{B_{j+1}}, \quad j \in\{0, \ldots, p-1\} .
$$

Similarly, $L_{p-1}=\lambda-1$, which is $U(1)$, and it follows that $L_{p-j}=U^{j}(1)$. Thus,

$$
L_{p-j}=\frac{B_{j+1}-B_{j}}{B_{j}-B_{j-1}}, \quad j \in\{1, \ldots, p-1\} .
$$

Hence,

$$
V=\prod_{k=1}^{p-1} \frac{1-\frac{B_{p-1-k}}{B_{p-k}} \cdot \frac{B_{p-k+1}-B_{p-k}}{B_{p-k}-B_{p-k-1}}}{1-\frac{B_{p-k}}{B_{p-k+1}} \cdot \frac{B_{p-k+1}-B_{p-k}}{B_{p-k}-B_{p-k-1}}}
$$

Clearing denominators, we get

$$
V=\prod_{k=1}^{p-1}\left(\frac{B_{p-k}^{2}-B_{p-k+1} B_{p-k-1}}{B_{p-k}^{2}-B_{p-k+1} B_{p-k-1}}\right) \cdot \prod_{k=1}^{p-1} \frac{B_{p-k+1}}{B_{p-k}} .
$$

This easily gives that $V=B_{p}$. The lemma hence follows.

Remark. The value of $C$ as given above is already implicit in work of H. Nakada [N2] on the even index Rosen fractions. Projecting $\nu$, we find the invariant measure, $\rho$, of $f$. The arguments of Nakada [N1] easily extend to the current setting so as to prove that the dynamical system $(I, \mathcal{B}, \rho, f)$ is ergodic, and even exact; thus $(\Omega, \mathcal{B}, \nu, \mathcal{T})$ is Kolmogorov. Moreover, in an upcoming section, we will prove that the dynamical system $(I, \mathcal{B}, \rho, f)$ is weak Bernoulli with respect to the natural partition.

3.2. Odd indices. We have the same goal as in the previous subsection, but we will find that constructing a natural extension for the case of an odd $q$ is a bit more involved. Now, fix an odd $q$ and recycle notation as above. Let $I$ be the interval $I:=\left[-\frac{\lambda}{2}, \frac{\lambda}{2}\right)$. As above, let $\phi_{j}=f^{j}(-\lambda / 2)$. With $h=\frac{q-3}{2}$, it follows from equation (4) that

$$
-\frac{\lambda}{2} \leq \phi_{j}<-\frac{2}{3 \lambda} \text { for } j \in\{0, \cdots, h-1\} \cup\{h+1, \cdots, 2 h\}
$$

and that

while $\phi_{2 h+1}=0$.

$$
-\frac{2}{3 \lambda}<\phi_{h}<-\frac{2}{5 \lambda}
$$



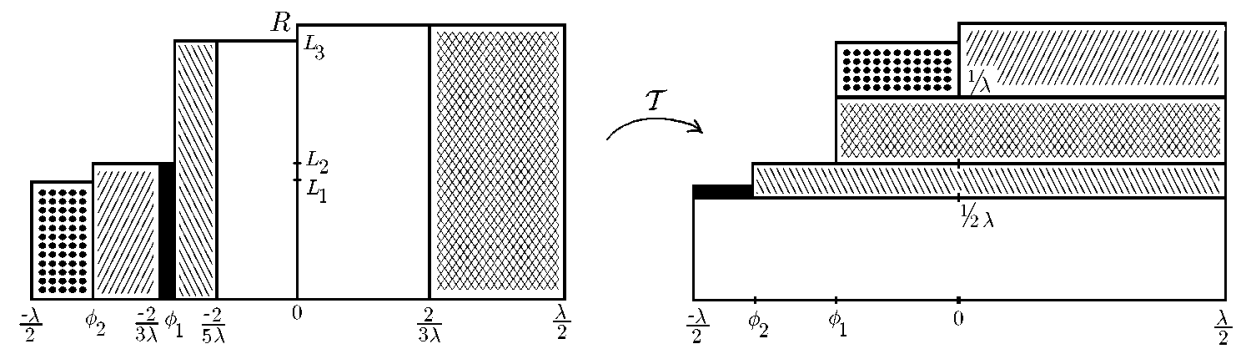

Figure $2 . \Omega$ and $\mathcal{T}(\Omega)$ for $q=5$.

We partition $I$ with $J_{j}, j \in\{1, \cdots, 2 h+2\}$, where

$$
\begin{aligned}
J_{2 k} & =\left[\phi_{h+k}, \phi_{k}\right), \text { for } k \in\{1, \cdots, h\}, \\
J_{2 k+1} & =\left[\phi_{k}, \phi_{h+k+1}\right), \text { for } k \in\{0,1, \cdots, h\},
\end{aligned}
$$

and $J_{2 h+2}=\left[0, \frac{\lambda}{2}\right)$. Let $K_{j}=\left[0, L_{j}\right]$ for $j \in\{1, \cdots, 2 h+1\}$ and let $K_{2 h+2}=[0, R]$. See also Figure 2.

Theorem 3.2. Let $q=2 h+3$, with $h \geq 1$ and $\Omega=\bigcup_{j=1}^{2 h+2} J_{j} \times K_{j}$. If the relations

$$
\left\{\begin{array}{ll}
\left(\mathcal{R}_{0}\right): & R=\lambda-L_{2 h+1}, \\
\left(\mathcal{R}_{1}\right): & L_{1}=1 /\left(2 \lambda-L_{2 h}\right), \\
\left(\mathcal{R}_{2}\right): & L_{2}=1 /\left(2 \lambda-L_{2 h+1}\right), \\
\left(\mathcal{R}_{j}\right): & L_{j}=1 /\left(\lambda-L_{j-2}\right) \\
\left(\mathcal{R}_{2 h+2}\right): & R=1 /\left(\lambda-L_{2 h}\right)
\end{array} \text { for } 2<j<2 h+2,\right.
$$

all hold, then $\mathcal{T}: \Omega \rightarrow \Omega$ is bijective off of a set of Lebesgue measure zero.

Proof. We proceed as for the case of even indices. The relation $\mathcal{R}_{0}$ ensures that $\mathcal{T}$ takes $(-2 / 5 \lambda, 0) \times\left[0, L_{2 h+1}\right] \bigcup(0,2 / 3 \lambda) \times[0, R]$ onto $I \times[0,1 / 2 \lambda]$. Note that $f(x)=-1 / x-2 \lambda$ for $x \in[-2 / 3 \lambda,-2 / 5 \lambda)$. By $\mathcal{R}_{1}, \mathcal{T}$ takes $[-2 / 3 \lambda,-2 / 5 \lambda) \times\left[0, L_{2 h}\right]$ onto $\left(\left[0, \phi_{h+1}\right) \times\left[1 / 2 \lambda, L_{1}\right]\right)=J_{1} \times\left[1 / 2 \lambda, L_{1}\right]$. By $\mathcal{R}_{1},\left[\phi_{h},-2 / 5 \lambda\right) \times\left[0, L_{2 h+1}\right]$ is taken to $\left[\phi_{h+1}, \lambda / 2\right) \times\left[1 / 2 \lambda, L_{2}\right]$.

For $x \in[-\lambda / 2,-2 / 3 \lambda), f(x)=-1 / x-\lambda=S^{-1} T(x)$. Thus, for $k \in\{1, \ldots, 2 h\}$, the relation $\mathcal{R}_{k+2}$ shows that $\mathcal{T}$ takes $J_{k} \times\left[0, L_{k}\right]$ to $J_{k+2} \times\left[1 / \lambda, L_{k+2}\right]$. Finally, by $\mathcal{R}_{2 h+2}, \mathcal{T}$ sends $\left[\phi_{2 h},-2 / 3 \lambda\right) \times\left[0, L_{2 h}\right]$ to $\left(\left[\phi_{2 h+1}, \lambda / 2\right) \times[1 / \lambda, R]\right)=J_{2 h+2} \times$ $[0, R]$.

We determine $R$. For periodic continued fraction expansions, we use an overline to indicate a period, and denote the sign which precedes the period by $(\epsilon:)$.

Lemma 3.3. For $q=2 h+3$, with $h \geq 1$, the system $\mathcal{R}_{0}, \mathcal{R}_{1}, \ldots, \mathcal{R}_{2 h+2}$ admits a unique real solution. For this solution, $R$ satisfies the equation

$$
R^{2}+(2-\lambda) R-1=0
$$

and the $\lambda C F$ expansion of $R$ is

$$
R=\lambda+\left[\overline{(-1: 1)^{h},-1: 2,(-1: 1)^{h-1},-1: 2,(-1:)}\right] .
$$

In particular, $1>R>\lambda / 2$. 
Proof. As above, the system shows that $R$ has a periodic expansion. Indeed, from $\mathcal{R}_{0}, \mathcal{R}_{2 h+1}, \mathcal{R}_{2 h-1}, \ldots, \mathcal{R}_{3}, \mathcal{R}_{1}$, on solving $\mathcal{R}_{2 h+2}$ for $L_{2 h}$, we find that

$$
R=\lambda-\frac{1}{\lambda-\ldots-\frac{1}{2 \lambda-L_{2 h}}}=(S T)^{h+2}(-R) .
$$

Similarly, $\mathcal{R}_{2 h+2}, \mathcal{R}_{2 h}, \ldots, \mathcal{R}_{2}$ and $\mathcal{R}_{0}$ give

$$
R=\left[\left(T S^{-1}\right)^{h+1}\right](-R) \text {. }
$$

Now, $U^{q}=(S T)^{q}$ acts as the identity; hence

$$
\left(T S^{-1}\right)^{h+1}=(S T)^{2 h+3}\left(T S^{-1}\right)^{h+1}=(S T)^{h+2} .
$$

Therefore, (A) and (B) are equivalent statements.

Note that $-T S^{-1}(-x)=T S(x)$. An easy induction argument gives us

$$
-\left(T S^{-1}\right)^{m}(-x)=(T S)^{m}(x) .
$$

Thus, (A) and (B) give us

$$
\begin{aligned}
R & =(S T)^{h+2}(-R)=(S T)^{h+2}(T S)^{h+1}(R) \\
& =(S T)^{h+1} S^{2}(T S)^{h+1}(R) \\
& =(S T)^{h+1} S^{2} T(S T)^{h}(\lambda+R) .
\end{aligned}
$$

Hence,

$$
\begin{aligned}
R-\lambda & =-L_{2 h+1} \\
& =(T S)^{h} \cdot T S^{2} \cdot(T S)^{h} \cdot T S^{2}\left(-L_{2 h+1}\right) .
\end{aligned}
$$

Therefore, the relations $\mathcal{R}_{0}$ through $\mathcal{R}_{2 h+2}$ imply that $-L_{2 h+1}$ has the purely periodic expansion $\left[\overline{(-1: 1)^{h},-1: 2,(-1: 1)^{h-1},-1: 2,(-1:)}\right]$. This proves uniqueness. Now, if $-L_{2 h+1}$ is of this form, then one easily checks that all of the relations $\mathcal{R}_{i}$ can indeed be satisfied.

Now, from (A) and (3),

$$
R=U^{h+2}(-R)=\frac{B_{h+3} R+B_{h+2}}{B_{h+2} R+B_{h+1}} .
$$

Since $U^{h}(\lambda)=1$, we find that $B_{h+2}=B_{h+1}$. The recursion relation for the $B_{n}$ now gives that $B_{h+3}=(\lambda-1) B_{h+1}$. Therefore,

$$
R=\frac{(\lambda-1) R+1}{R+1}
$$

The quadratic equation is now evident.

The expansion for $R$ shows that $R=U^{h}(\lambda-1 /(2 \lambda-\ldots))$. Since $U(x)$ is increasing, $R<U^{h}(\lambda)=1$. Similarly, $R>U^{h+1} S^{2}(T S)^{h-1}(\lambda)=U(2 / \lambda)=$ $\lambda / 2$.

As in the even case, we see that for $q$ odd, $\mathcal{T}$ is bijective on $\Omega$. A similar Jacobian calculation shows that $\mathcal{T}$ preserves the probability measure $\nu$ with density

$$
\frac{C}{(1+x y)^{2}} \text {, }
$$

where $C$ is a normalizing constant. We have the following lemma.

Lemma 3.4. Let $q$ be odd. The normalizing constant $C$ such that $\nu$ is a probability measure on $\Omega$ is given by

$$
C=1 / \ln (1+R)
$$


Proof. Integration gives

$$
C^{-1}=\ln W
$$

where

$$
W:=\prod_{j=0}^{h} \frac{1+L_{2 h+1-2 j} \cdot \phi_{2 h+1-j}}{1+L_{2 h+1-2 j} \cdot \phi_{h-j}} \cdot \prod_{j=0}^{h-1} \frac{1+L_{2(h-j)} \cdot \phi_{h-j}}{1+L_{2(h-j)} \cdot \phi_{2 h-j}} \cdot(1+\lambda / 2) .
$$

As above, $\phi_{2 h+1}=0$ and $\phi_{2 h}=-1 / \lambda$; indeed,

$$
\phi_{2 h+1-j}=-\frac{B_{j}}{B_{j+1}}, \quad j \in\{0, \ldots, h\} .
$$

Recall that $U^{h}(\lambda)=1$. From this, $\phi_{h}=-1 /\left(2 \lambda-\phi_{h+1}\right)$ gives is $\phi_{h}=-1 /(\lambda+1)$. It now follows that $\phi_{h-j}=(T S)^{j+1}(1)$, or

$$
\phi_{h-j}=\frac{-\left(B_{j}+B_{j+1}\right)}{B_{j+1}+B_{j+2}}, \quad j \in\{0, \ldots, h\} .
$$

Similarly, $L_{2 h}=U(R)$, and it follows that

$$
L_{2(h-j)}=\frac{B_{j+2} R-B_{j+1}}{B_{j+1} R-B_{j}}, \quad j \in\{0, \ldots, h-1\} .
$$

Also, from $L_{2 h+1}=U(1 / R)$, we have

$$
L_{2 h+1-2 j}=U^{j+1}(1 / R)=\frac{B_{j+1} R-B_{j+2}}{B_{j} R-B_{j+1}}, \quad j \in\{0, \ldots, h\} .
$$

We consider the different types of factors of $W$ separately. First, we have

$$
\frac{1+L_{2 h+1-2 j} \cdot \phi_{2 h+1-j}}{1+L_{2 h+1-2 j} \cdot \phi_{h-j}}=\frac{1-\frac{B_{j+1} R-B_{j+2}}{B_{j} R-B_{j+1}} \cdot \frac{B_{j}}{B_{j+1}}}{1-\frac{B_{j+1} R-B_{j+2}}{B_{j} R-B_{j+1}} \cdot \frac{B_{j}+B_{j+1}}{B_{j+1}+B_{j+2}}} .
$$

Clearing denominators, we find that

$$
\frac{1+L_{2 h+1-2 j} \cdot \phi_{2 h+1-j}}{1+L_{2 h+1-2 j} \cdot \phi_{h-j}}=\left(\frac{B_{j+1}+B_{j+2}}{B_{j+2}}\right) \cdot(1+R)^{-1} .
$$

In a similar fashion,

$$
\frac{1+L_{2(h-j)} \cdot \phi_{h-j}}{1+L_{2(h-j)} \cdot \phi_{2 h-j}}=\left(\frac{B_{j+1}+B_{j+2}}{B_{j+2}}\right)^{-1} \cdot(1+R) .
$$

Now, from the expansion of 1 we get $B_{h+2}=B_{h+1}$, and therefore

$$
W=\frac{2}{1+R} \cdot \frac{2+\lambda R}{2} .
$$

But, by use of the quadratic relation which $R$ satisfies,

$$
(1+R)^{2}=2+\lambda R .
$$

The lemma now follows.

Remark. Again, the marginal measure for $\nu$ gives the invariant measure $\rho$ for $f$, and arguments of Nakada [N1] can be shown to apply in the current setting, so as to prove that the dynamical system $(I, \mathcal{B}, \rho, f)$ is ergodic, and even exact; thus $(\Omega, \mathcal{B}, \nu, \mathcal{T})$ is Kolmogorov. Moreover, in the last section, we will prove that the dynamical system $(I, \mathcal{B}, \rho, f)$ is weak Bernoulli. 


\section{Arithmetic AND metric Results}

In this section we obtain a number of arithmetic and metric results by applying a method similar to that of $[\mathrm{JK}]$ and $[\mathrm{K} 1],[\mathrm{K} 2]$. We generalize and improve upon various results of Lehner [L2] and Nakada [N2].

Let some index $q$ be fixed. The approximation coefficients $\Theta_{n}=\Theta_{n}(x), n \geq 0$, for $x \in \mathbf{R}$ are given by

$$
\Theta_{n}=\Theta_{n}(x):=q_{n}^{2}\left|x-\frac{p_{n}}{q_{n}}\right|, n \geq 0,
$$

where $p_{n} / q_{n}$ is the $n$th $\lambda_{q} \mathrm{CF}$-convergent. Define $\mu_{q}(x)$ as the infimum of the $\Theta_{n}(x)$, and the Hurwitz constant $h_{q}:=\sup \left\{\mu_{q}(x) \mid x\right.$ is $G_{q}$-irrational $\}$. One has the following theorem, where we write $R_{q}$ to stress the dependence of $R$ upon $q$.

Theorem 4.1 (Haas-Series, Lehner). For all $q \geq 3, h_{q}=\mu_{q}\left(R_{q}\right)$.

Proof. (Sketch) This easily follows from [L1], [HS], [L2]. However, one can also adjust the argument of [JK] (Theorem 5.9) with the remaining material in this section to give an elementary geometric proof.

Remarks. (1) Originally, Lehner [L1] obtained $h_{q}$ for even $q$ by clever elementary continued fractions methods. It was left for Haas and Series [HS] to use non-trivial hyperbolic geometric techniques to determine $h_{q}$ for odd $q$, which Lehner [L2] then verified with his own techniques.

(2) We will return to our proof in a forthcoming paper.

We now examine bounds on the coefficients $\Theta_{n}$. Let $\left(t_{n}, v_{n}\right)=\mathcal{T}^{n}(x, 0)$ for $x=\left[\varepsilon_{1}: r_{1}, \varepsilon_{2}: r_{2}, \cdots, \varepsilon_{n}: r_{n}, \cdots\right]$; then from [K2](Lemma (1.24)), we have

$$
\Theta_{n-1}=\frac{v_{n}}{1+t_{n} v_{n}}, \Theta_{n}=\frac{\varepsilon_{n+1} t_{n}}{1+t_{n} v_{n}}, n \geq 1
$$

As in [JK], [K1] and [K2], $\S 6$, this leads to a map $\psi: \Omega_{q} \rightarrow \mathbf{R}^{2}$, defined by

Clearly one has

$$
\psi(t, v):=\left(\frac{v}{1+t v}, \frac{|t|}{1+t v}\right) .
$$

$$
\psi\left(t_{n}, v_{n}\right)=\left(\Theta_{n-1}, \Theta_{n}\right) \text { for } n \geq 1
$$

and from

$$
\left(\Theta_{n}, \Theta_{n+1}\right)=\psi\left(\mathcal{T}\left(\psi^{-1}\left(\Theta_{n-1}, \Theta_{n}\right)\right)\right),
$$

one easily derives (compare with [JK], Theorem 5.8) that

$$
\Theta_{n+1}=\varepsilon_{n+2}\left(\varepsilon_{n+1} \Theta_{n-1}-\left(\lambda r_{n+1}\right)^{2} \Theta_{n}+\lambda r_{n+1} \sqrt{1-4 \varepsilon_{n+1} \Theta_{n-1} \Theta_{n}}\right), n \geq 1 .
$$

Putting

$$
\Gamma_{q}:=\psi\left(\Omega_{q}\right)
$$

and

$$
\Gamma_{q}^{+}:=\psi\left(\Omega_{q}^{+}\right), \Gamma_{q}^{-}:=\psi\left(\Omega_{q}^{-}\right)
$$

where

$$
\Omega_{q}^{+}:=\left\{(t, v) \in \Omega_{q} \mid t \geq 0\right\} ; \Omega_{q}^{-}:=\left\{(t, v) \in \Omega_{q} \mid t<0\right\}
$$


one obviously has that

$$
\begin{aligned}
& \varepsilon_{n+1}=+1 \Rightarrow\left(\Theta_{n-1}, \Theta_{n}\right)=\psi\left(t_{n}, v_{n}\right) \in \Gamma_{q}^{+}, \\
& \varepsilon_{n+1}=-1 \Rightarrow\left(\Theta_{n-1}, \Theta_{n}\right)=\psi\left(t_{n}, v_{n}\right) \in \Gamma_{q}^{-} .
\end{aligned}
$$

4.1. The positive region, $\Gamma_{q}^{+}$. The region $\Gamma_{q}^{+}$can easily be described. For all $q \geq 3, \Gamma_{q}^{+}$is a quadrangle with vertices

$$
(R, 0),(0,0),\left(\frac{\lambda}{2}, 0\right) \text { and }\left(\frac{2 R}{2+\lambda R}, \frac{\lambda}{2+\lambda R}\right) .
$$

Thus, $\Gamma_{q}^{+}$is bounded by the positive coordinate axes and by line segments of equation $y=-\left(\lambda^{2} / 4\right) x+\lambda / 2$ and $y=-x+1$. See Figure 3 .

Horizontal lines of equation $t=K$ in $\Omega_{q}^{+}$(for $K$ a positive constant) are mapped under $\psi$ to lines $y=-K^{2} x+K$ in $\Gamma_{q}^{+}$; vertical lines $v=K$ are mapped to lines $y=-\frac{1}{K^{2}} x+\frac{1}{K}$. Furthermore one has

$$
\frac{R}{R^{2}+1}<\frac{2 R}{\lambda R+2}
$$

which is obvious in case $q$ is even (since then $R=1$ ), and which follows in the odd case since $\lambda<2 R$, by Lemma 3.3.

Putting $V(\xi):=\left\{(x, y) \in \mathbf{R}^{2} \mid \min (x, y) \geq \xi\right\}$ for $\xi \in \mathbf{R}$, one has that

$$
\inf _{\xi \in \mathbf{R}}\left(V(\xi) \cap \Gamma_{q}^{+}=\emptyset\right)=\frac{2 \lambda}{\lambda^{2}+4} .
$$

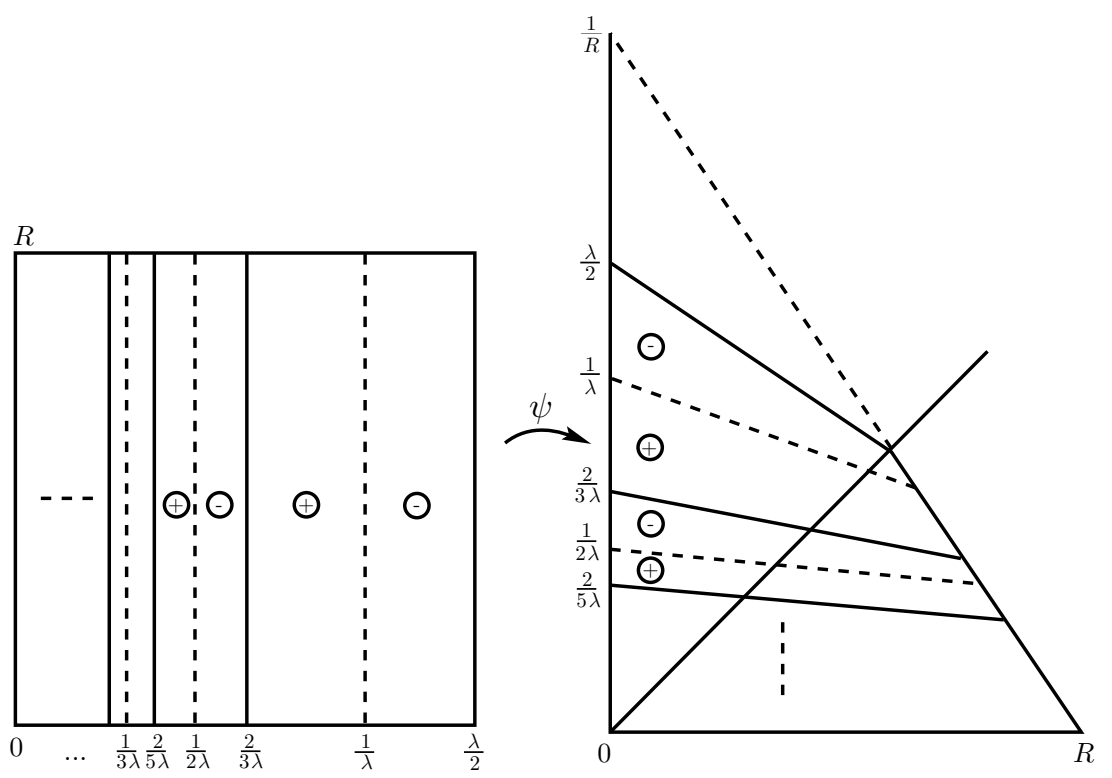

FiguRE 3. $\Gamma_{q}^{+}$ 
This immediately gives the following Vahlen-type theorem, generalizing Lehner's [L2, Theorem 2].

Theorem 4.2. For all $q \geq 3$, for all $G_{q}$-irrational numbers $x$ and all positive integers $n$ for which $\varepsilon_{n+1}(x)=+1$, one has

$$
\max \left(\Theta_{n-1}(x), \Theta_{n}(x)\right) \leq R \text { and } \min \left(\Theta_{n-1}(x), \Theta_{n}(x)\right) \leq \frac{2 \lambda}{\lambda^{2}+4}
$$

In case $\varepsilon_{n+1}(x)=\varepsilon_{n+2}(x)=+1$ one easily finds that for $q>3$ the same idea yields $\min \left(\Theta_{n-1}(x), \Theta_{n}(x)\right) \leq \lambda /\left(\lambda^{2}+1\right)$. We refine this result further. Recall that $\Delta[+1: r]$ is the set of those $x$ in $I_{q}$ for which the expansion begins with $1 / r$. A simple calculation shows that

$$
\Delta[+1: r]= \begin{cases}\left(\frac{2}{3 \lambda}, \frac{\lambda}{2}\right), & \text { if } r=1, \\ \left(\frac{2}{(2 r+1) \lambda}, \frac{2}{(2 r-1) \lambda}\right), & \text { if } r \geq 2\end{cases}
$$

and note that $\Delta[+1: r]=\Delta_{[+1: r,+1:]} \cup \Delta_{[+1: r,-1:]}$, where

$$
\Delta_{[+1: r,+1:]}=\left(\frac{2}{(2 r+1)}, \frac{1}{\lambda}\right), \Delta_{[+1: r,-1:]}=\Delta_{[+1: r]} \backslash \Delta_{[+1: r,+1:]},
$$

and that one has for $\varepsilon \in\{-1,+1\}$,

$$
\left(t_{n}, v_{n}\right) \in \Delta_{[+1: r, \varepsilon:]} \times[0, R] \Leftrightarrow \varepsilon_{n+1}=+1, \varepsilon_{n+2}=\varepsilon, r_{n+1}=r .
$$

Now every strip $\Delta_{[+1: r,+1:]} \times[0, R]$ has a unique fixed point $\left(\xi_{r}, \xi_{r}\right)$ under $\mathcal{T}$, and easy calculations show that

$$
\xi_{r}=\frac{-r \lambda+\sqrt{(r \lambda)^{2}+4}}{2}=[\overline{+1: r}]
$$

and

$$
\psi\left(\xi_{r}, \xi_{r}\right)=\left(\eta_{r}, \eta_{r}\right)
$$

where

$$
\eta_{r}=\frac{1}{\sqrt{(r \lambda)^{2}+4}}
$$

In the above, $[\overline{+1: r}]$ indicates the purely periodic expansion with all $\varepsilon=+1$ and all $r_{i}=r$.

In view of $(6)$, we define the map $s(x, y)$ on $\psi\left(\Delta_{[+1: r,+1:]} \times[0, R]\right)$ by

$$
s(x, y):=x-(r \lambda)^{2} y+r \lambda \sqrt{1-4 x y}
$$

(recall that on $\Delta_{[+1: r,+1:]} \times[0, R]$ one has $\varepsilon_{n+1}=\varepsilon_{n+2}=+1$ ).

Since

$$
\frac{\partial s}{\partial x}<0, \frac{\partial s}{\partial y}<0
$$

(the first inequality follows after some elementary calculations, the second is immediate, see also [JK], p. 292), and

$$
s\left(\frac{1}{\sqrt{(r \lambda)^{2}+4}}, \frac{1}{\sqrt{(r \lambda)^{2}+4}}\right)=\frac{1}{\sqrt{(r \lambda)^{2}+4}}
$$

the following theorem - which again generalizes a theorem of [L2] - follows directly (see also Figure 4). 


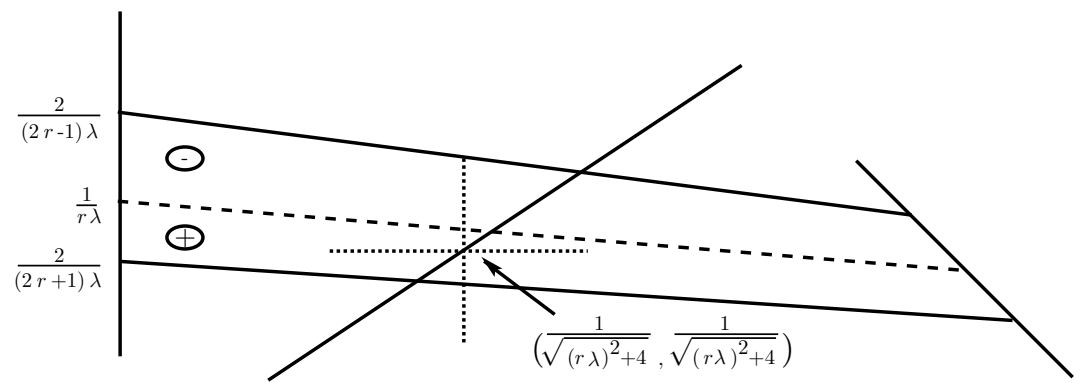

FiguRE 4. $\mathcal{T}\left(\Delta_{[+1: r, \varepsilon:]} \times[0, R]\right)$.

Theorem 4.3. For all $q \geq 3$, for all $G_{q}$-irrational numbers $x$ and all positive integers $n$ for which $\varepsilon_{n+1}(x)=\varepsilon_{n+2}(x)=+1$, one has

$$
\min \left(\Theta_{n-1}(x), \Theta_{n}(x), \Theta_{n+1}(x)\right) \leq \frac{1}{\sqrt{\left(r_{n+1} \lambda\right)^{2}+4}}
$$

and

$$
\max \left(\Theta_{n-1}(x), \Theta_{n}(x), \Theta_{n+1}(x)\right) \geq \frac{1}{\sqrt{\left(r_{n+1} \lambda\right)^{2}+4}} .
$$

Remark. Simple calculations show that in general the condition $\varepsilon_{n+2}=+1$ in Theorem 4.3 cannot be omitted.

4.2. The negative region, $\Gamma_{q}^{-}$. It is significantly harder to give general results for all $\Gamma_{q}^{-}$. Clearly each $\Omega_{q}^{-}$is a staircase-like domain and for any given $q \geq 3$, one can easily determine $\Gamma_{q}^{-}$. However, the fine detail of this structure greatly depends upon the parity of $q$, as we now discuss.

4.2.1. Even indices: $q=2 p$. In this setting, we have

$$
\psi\left(\phi_{j}, L_{j+1}\right)=\psi\left(\mathcal{T}\left(\phi_{j-1}, L_{j}\right)\right), j \in\{1,2, \ldots, p-1\} .
$$

Putting

$$
\left(\theta_{0}, \theta_{1}\right):=\psi\left(\phi_{0}, L_{1}\right)=\left(\frac{2}{\lambda+2}, \frac{\lambda(\lambda+1)}{\lambda+2}\right)
$$

we see that

$$
\psi\left(\phi_{1}, L_{2}\right)=\psi\left(\mathcal{T}\left(\psi^{-1}\left(\theta_{0}, \theta_{1}\right)\right)\right)=\left(\theta_{1}, s\left(\theta_{0}, \theta_{1}\right)\right),
$$

where $s(\alpha, \beta)=\alpha+\lambda^{2} \beta-\lambda \sqrt{1+4 \alpha \beta}$, as in (6). In view of this, for $j \in$ $\{1,2, \ldots, p-1\}$ recursively define the numbers $\theta_{j}$ by

$$
\theta_{j+1}=s\left(\theta_{j-1}, \theta_{j}\right) \text {. }
$$

Clearly one has

$$
\psi\left(\phi_{j-1}, L_{j}\right)=\left(\theta_{j-1}, \theta_{j}\right), j \in\{1,2, \ldots, p-1\} .
$$

Notice furthermore that $\left(\theta_{p-2}, \theta_{p-1}\right)=(\lambda(\lambda-1), 1)$ - see also Figure 5 .

From the above, we find that the rectangle in $\Omega^{-}$with endpoints $\left(\phi_{j-1}, 0\right),(0,0)$, $\left(0, L_{j}\right)$ and $\left(\phi_{j-1}, L_{j}\right)$ is bijectively mapped by $\psi$ onto the quadrangle $\mathcal{Q}_{j}$ of vertices $\left(0,\left|\phi_{j-1}\right|\right),(0,0),\left(L_{j}, 0\right)$ and $\left(\theta_{j-1}, \theta_{j}\right)$, where $j \in\{1,2, \ldots, p-1\}$. The union of 


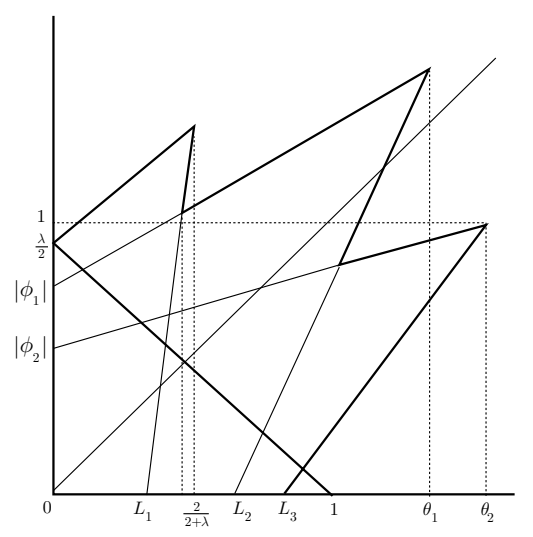

FIGURE $5 . \Gamma_{q}^{+}$and $\Gamma_{q}^{-}$for $q=8$.

these quadrangles is $\Gamma_{q}^{-}$.

The sequence $\left|\phi_{j-1}\right|$ decreases monotonically, while the sequence $L_{j}$ increases monotonically. We study the relationship between these values in the following lemma.

Lemma 4.1. Let $j^{*}=\left\lfloor\frac{q}{4}\right\rfloor$. Then

$$
\left\{\begin{array}{l}
\left|\phi_{j-1}\right|>L_{j} \quad \text { if } 1 \leq j \leq j^{*} \\
\left|\phi_{j-1}\right|<L_{j} \quad \text { if } j^{*}<j<p .
\end{array}\right.
$$

Proof. For any index $q$, it is easily shown that the values $\lambda=U^{0}(\lambda)>U^{1}(\lambda)>$ $\cdots>U^{q-2}(\lambda)=0$ lie symmetrically about the line $x=\lambda / 2$. When $q=2 p$, this implies that $\lambda / 2=U^{p-1}(\lambda)$. But, $\left|\phi_{0}\right|=\lambda / 2$ and the $\left|\phi_{j}\right|$ are images of this under powers of $U$. Thus,

$$
\left|\phi_{j-1}\right|=U^{p+j-2}(\lambda) \text { for } j \in\{1, \ldots, p\} .
$$

We already have, by the sentence preceding equation (5), that $L_{j}=U^{p-j}(1)$ for $j \in\{1, \ldots, p-1\}$. Of course, $U(x)=\lambda-1 / x$ increases with increasing $\lambda$; thus, from the expansion of 1 for odd indices, see the sentence following (4), we deduce that for $q=2 p$,

$$
U^{p-1}(\lambda)<1<U^{p-2}(\lambda)
$$

Now, as $U(x)$ is strictly increasing in $x$,

$$
\left|\phi_{j-1}\right|<U^{j-1}(1)<\left|\phi_{j-2}\right|
$$

and thus

$$
U^{j}(1)<\left|\phi_{j-1}\right|<U^{j-1}(1)
$$

Hence, $\left|\phi_{j-1}\right|>L_{j}$ holds if and only if $p-j \geq j$. This immediately gives our lemma.

The following Vahlen-type theorem follows almost directly from the above lemma. It is a strengthening of theorems found in [L2]. 
Theorem 4.4. Let $q=2 p$ be an even integer greater than or equal to $4, j^{*}=\left\lfloor\frac{q}{4}\right\rfloor$ and let

$$
\left\{\begin{array}{l}
\mathcal{V}_{2 l}=\max \left\{\theta_{j^{*}-l}, \theta_{j^{*}+l}\right\}, \quad \text { for } l \geq 1, \\
\mathcal{V}_{2 l+1}=\min \left\{\theta_{j^{*}-l}, \theta_{j^{*}+l}\right\},
\end{array}\right.
$$

Then for all $G_{q}$-irrational numbers $x$ and all $n \geq 1$ one has

(i) $0<\min \left\{\Theta_{n}(x), \Theta_{n+1}(x), \ldots, \Theta_{n+k-1}(x)\right\} \leq \mathcal{V}_{k}$, for $1 \leq k \leq p-1$;

$$
0<\sum_{j=0}^{k-1} \Theta_{n+j}(x) \leq \sum_{j=1}^{k} \mathcal{V}_{j}, \quad \text { for } 1 \leq k \leq p-1 ;
$$

(iii) $-\frac{\lambda}{2}<\Theta_{n-1}(x)-\Theta_{n}(x)<1$.

Proof. From the definition of $\psi$, Lemma 4.1 implies that $\theta_{j-1}<\theta_{j}$ for all $j \leq j^{*}$, and $\theta_{j-1}>\theta_{j}$ for all $j>j^{*}$. Now, for $x$ and $n$ as above, $\left(\Theta_{n-1}(x), \Theta_{n}(x)\right)$ lies in $\Gamma_{q}$. Hence, the maximum value of either of these coordinates is given by the maximum of the coordinates of the vertices of the polygonal region which $\Gamma_{q}$ forms. Since $\theta_{j^{*}}$ is greater than any coordinate in $\Gamma_{q}^{+}$, the first of our inequalities follows when $k=1$.

In the general case, we note that if some $\Theta_{m}(x)$ is large, so that there is a $j<p-1$ with $\left(\Theta_{m}(x), \Theta_{m+1}(x)\right)$ being in $\mathcal{Q}_{j}$, then $\left(\Theta_{m+1}(x), \Theta_{m+2}(x)\right)$ must be in $\mathcal{Q}_{j+1}$. Again, since all coordinates of points in $\Gamma_{q}^{+}$are smaller than $1=\theta_{p-1},(i)$ follows.

Related reasoning gives the rest of the theorem.

Remarks. (1) Using the definitions, one shows that $\theta_{j}=\left(B_{p-j}-B_{p-j-1}\right) B_{p-j}$. (2) Specific values of $\mathcal{V}_{1}=\theta_{j^{*}}$ are as follows: $\left(q=8, \theta_{j^{*}}=1.56645 \ldots\right) ;(q=80$, $\left.\theta_{j^{*}}=12.9858 \ldots\right) ;\left(q=8000, \theta_{j^{*}}=1273.49 \ldots\right)$.

(3) Numerical evidence seems to indicate that $\mathcal{V}_{2}$ being $\theta_{j^{*}-1}$ or $\theta_{j^{*}+1}$ depends on the congruence class of $q$ modulo 4 . It appears that if 4 divides $q$, the first holds and otherwise, the second. We leave such fine points for the interested reader, who may wish to consider (6).

(4) Also we note that, in the limiting case (for both even and odd values of $q$ ), $\Gamma_{q \rightarrow \infty}^{-}$is bounded by the lines $y=x \pm 1$; it is easily seen that $\theta_{0}$ has a limit of $1 / 2$. But, the images under $\psi$ of the various vertical lines of $x$-cordinate $x=\phi_{j}$ tend to the line $y=x+1$. Thus, in the limit as $q$ goes to infinity, $\theta_{j}$ tends to $1 / 2+j$ for $j \leq h$ and similarly for the values of the other $\theta_{n}$.

4.2.2. Odd indices: $q=2 h+3$. Once again, the setting of odd indices is more complicated. As usual, we fix an odd $q$ and let $h=(q-3) / 2$. The following is the analog of the above lemma.

Lemma 4.2. Let $j^{*}=\left\lfloor\frac{h}{2}\right\rfloor$. Then

$$
\left\{\begin{array}{l}
\left|\phi_{j}\right|>L_{2 j+1} \quad \text { if } 0 \leq j \leq j^{*}, \\
\left|\phi_{j}\right|<L_{2 j+1} \quad \text { if } j^{*}<j<h .
\end{array}\right.
$$

Furthermore, if $k^{*}=\left\lceil\frac{h}{2}\right\rceil$, then

$$
\left\{\begin{array}{l}
\left|\phi_{h+j}\right|>L_{2 j} \quad \text { if } 1 \leq j \leq k^{*}, \\
\left|\phi_{h+j}\right|<L_{2 j+1} \quad \text { if } k^{*}<j<h .
\end{array}\right.
$$


Proof. For the first of these, we begin with $\lambda / 2<R$. Hence, $\lambda-R=U(1 / R)<\lambda / 2$. And, as $R<1$, we find that $1 / R>\lambda / 2>U(1 / R)$. Now, $\left|\phi_{j}\right|=U^{j}(\lambda / 2)$; hence, $U^{j}(1 / R)>\left|\phi_{j}\right|>U^{j+1}(1 / R)$. But, $L_{2 j+1}=U^{h+1-j}(1 / R)$. Thus, the conclusion follows.

For the second, from $\lambda / 2<R<1$ it follows that $U^{h+1}(\lambda)<R<U^{h}(\lambda)$. Since $L_{2 j}=U^{h+1-j}(R)$, we have

$$
U^{2 h+2-j}(\lambda)<L_{2 j}<U^{2 h+1-j}(\lambda) .
$$

But, $\left|\phi_{h+j}\right|=U^{h+j}$, and we are done with the proof of the lemma.

We now state the analog of Theorem 4.4.

Theorem 4.5. Let $q$ be an odd integer greater than or equal to $3, j^{*}=\left\lfloor\frac{q-3}{4}\right\rfloor$ and $k^{*}=\left\lceil\frac{q-3}{4}\right\rceil$. Further, let $\mathcal{W}_{k}$ be the $k$-th largest $\theta_{j}$. Then $\mathcal{W}_{1} \in\left\{\theta_{j^{*}+1}, \theta_{h+1+k^{*}}\right\}$. Furthermore, for all $G_{q}$-irrational numbers $x$ and all $n \geq 1$ one has

$$
0<\min \left\{\Theta_{n}(x), \Theta_{n+1}(x), \ldots, \Theta_{n+k-1}(x)\right\} \leq \mathcal{W}_{k}, \text { for } 1 \leq k \leq q-2 .
$$

Proof. Using the definition of $\theta_{1}$ and the equation which $R$ satisfies, one easily verifies that $\theta_{1}>R$. Hence the theorem follows from Lemma 4.2 and the description of the natural extensions.

Remark. Numerical evidence seems to indicate that $\mathcal{W}_{1}$ is in fact always $\theta_{j^{*}+1}$. As to limiting values of the $\theta_{j}$, see the previous remark.

4.3. On the distribution of the $\Theta_{n}$. Since the dynamical system $(\Omega, \mathcal{B}, \nu, \mathcal{T})$ is ergodic, the following proposition follows almost directly; see [K1], Theorem 5.

Proposition 4.1. Let $q \geq 3$. For almost all $G_{q}$-irrational numbers $x$, the twodimensional sequence

$$
\left(f_{q}^{n}(x), \frac{q_{n-1}}{q_{n}}\right)_{n \geq 1}
$$

is distributed over $\Omega_{q}$ according to the density function $g_{q}$ given by

$$
g_{q}(t, v)= \begin{cases}\frac{C}{(1+t v)^{2}}, & \text { for }(t, v) \in \Omega_{q}, \\ 0, & \text { otherwise. }\end{cases}
$$

Here $C$ is the normalizing constant of the $\mathcal{T}$-invariant measure $\rho$. See Lemma 3.2 (for q even) and Lemma 3.4 ( $q$ odd).

Remark. The sequence $\left(f_{q}^{n}(x), \frac{q_{n-1}}{q_{n}}\right)$ is rather special; indeed the $G_{q}$-irrationals being of measure zero means that this sequence lies within a two-dimensional measure zero set. However, the above proposition shows that almost always the sequence is distributed just as a generic sequence.

Now consider the map

$$
F:(t, v) \mapsto\left(\frac{v}{1+t v}, \frac{t}{1+t v}\right)=:(\alpha, \beta), \text { for } t v \neq-1 .
$$

It is easily verified that $F$ maps $\Omega_{q}$ onto $\Gamma_{q}^{+} \cup \Gamma_{q}^{*}$, where $\Gamma_{q}^{*}$ is the reflection of $\Gamma_{q}^{-}$ in the $\alpha$-axis. For the derivative $F^{\prime}$ of $F$ we have

$$
F^{\prime}(t, v)=\left[\begin{array}{cc}
\frac{-v^{2}}{(1+t v)^{2}} & \frac{1}{(1+t v)^{2}} \\
\frac{1}{(1+t v)^{2}} & \frac{-t^{2}}{(1+t v)^{2}}
\end{array}\right]
$$


and therefore the Jacobi determinant $J$ of $F$ equals $-(1-t v)(1+t v)^{-3}$. From this and Proposition 4.1 it now follows that for almost all $x$ the sequence

$$
\left(\left(F\left(t_{n}, v_{n}\right)\right)=\left(F\left(f_{q}^{n}(x), \frac{q_{n-1}}{q_{n}}\right)\right), \quad n=1,2, \ldots,\right.
$$

which is just the sequence $\left(\Theta_{n-1}(x), \varepsilon_{n+1} \Theta_{n}(x)\right), n=1,2, \ldots$, is distributed on $\Gamma_{q}^{+} \cup \Gamma_{q}^{*}$ according to the density function $g_{q}|J|^{-1}$.

Since

$$
\begin{aligned}
& g_{q}|J|^{-1}=C\left|\frac{1+t v}{1-t v}\right|=C\left(\left(\frac{1-t v}{1+t v}\right)^{2}\right)^{-1 / 2} \\
& =C\left(\frac{(1+t v)^{2}-4 t v}{(1+t v)^{2}}\right)^{-1 / 2}=\frac{C}{\sqrt{1-4 \alpha \beta}}
\end{aligned}
$$

we have obtained the following theorem.

Theorem 4.6. Let $q \geq 3$ and define the functions $d_{q}^{+}$and $d_{q}^{-}$by

$$
d_{q}^{+}(\alpha, \beta):= \begin{cases}\frac{C}{\sqrt{1-4 \alpha \beta}}, & \text { for }(\alpha, \beta) \in \Gamma_{q}^{+}, \\ 0, & \text { otherwise }\end{cases}
$$

and

$$
d_{q}^{-}(\alpha, \beta):= \begin{cases}\frac{C}{\sqrt{1+4 \alpha \beta}}, & \text { for }(\alpha, \beta) \in \Gamma_{q}^{-}, \\ 0, & \text { otherwise. }\end{cases}
$$

Then for all $G_{q}$-irrational numbers $x$, the sequence $\left(\Theta_{n-1}(x), \Theta_{n}(x)\right), n=1,2, \ldots$, is distributed over the interior of $\Gamma_{q}$, and for almost all $x$ this distribution is according to the density function $d_{q}$, where

$$
d_{q}(\alpha, \beta):=d_{q}^{+}(\alpha, \beta)+d_{q}^{-}(\alpha, \beta) .
$$

Notice that an alternative form of the second part of Theorem 4.6 can be stated as follows.

Theorem 4.6' Let $q \geq 3$ and let $d_{q}$ be defined as in Theorem 4.6. Then for almost all $x$ and for every $a, b$ with $a, b \geq 0$ the limit

$$
\lim _{N \rightarrow \infty} \frac{1}{N} \#\left\{j ; 1 \leq j \leq N, \Theta_{j-1}(x)<a, \Theta_{j}(x)<b\right\}
$$

exists, and equals

$$
\int_{0}^{a} \int_{0}^{b} d_{q}(\alpha, \beta) d \alpha d \beta
$$

Several corollaries can be drawn from Theorem 4.6, see e.g. [K1], where (for $q=3)$ for almost all $x$ the distributions of the sequences $\left(\Theta_{n}\right)_{n \geq 1},\left(\Theta_{n-1}+\Theta_{n}\right)_{n \geq 1}$, and $\left(\Theta_{n-1}-\Theta_{n}\right)_{n \geq 1}$ were determined. Here we only mention the following corollary, which was previously obtained by Nakada [N2] under the restriction that $q$ be even. The proof is left to the reader. 
Corollary 4.1. Let $q \geq 3$ and let $\mathcal{L}_{q}$ be defined by

$$
\mathcal{L}_{q}:= \begin{cases}\frac{\lambda}{\lambda+2}, & \text { q even }, \\ \frac{R}{R+1}, & \text { q odd },\end{cases}
$$

where $C$ and $R$ are defined as above. Then, for almost all $G_{q}$-irrational numbers $x$ and all $c \geq 1 / \mathcal{L}_{q}$,

$$
\lim _{N \rightarrow \infty} \frac{1}{N} \#\left\{n: 1 \leq n \leq N, \Theta_{n}(x)<\frac{1}{c}\right\}=\frac{\lambda C}{c} .
$$

\section{QUALITY OF MIXING}

In this section we will show that the Rosen $\lambda$ CF's are weak Bernoulli for $q \geq 4$ (the case $q=3$ can be treated by adapting the method). In [R], G.J. Rieger showed that the nearest integer continued fraction (which is our case $q=3$ ) has mixing properties even stronger than weak Bernoulli.

Fix $q \geq 4$ and let $I, f$ and $\rho$ be as above. Throughout this section, we will use an abbreviated notation for cylinder sets by writing $\left[a_{1}, a_{2}, \ldots, a_{m}\right]$ for the cylinder set $\Delta\left[\varepsilon_{1}:\left|a_{1}\right|, \varepsilon_{2}:\left|a_{2}\right|, \ldots, \varepsilon_{m}:\left|a_{m}\right|\right]$, where $a_{i}=\varepsilon_{i} \cdot\left|a_{i}\right|$.

The dynamical system $(I, f, \rho)$ has a natural partition $\mathcal{P}=\left\{P_{n}: n \in \mathbf{Z} \backslash\{0\}\right\}$ given by $P_{n}=[n]$.

Let

$$
l(q)= \begin{cases}q / 2-1 & \text { if } q \text { is even } \\ (q-3) / 2 & \text { if } q \text { is odd }\end{cases}
$$

A cylinder $\left[a_{0}, a_{1}, \ldots, a_{m}\right]$ has positive $\rho$-measure if and only if the string $a_{0}, a_{1}$, $\ldots, a_{m}$ contains no forbidden (that is, non-admissible) substrings; compare Figure 1. For even $q$, there are three types of forbidden substrings:

(i) $l(q)+1$ consecutive occurrences of -1 ;

(ii) $l(q)$ consecutive occurrences of -1 following a 1 ;

(iii) a $k$ following $l(q)$ consecutive occurrences of -1 , for any $k<-1$.

Note that this implies that if $k>1$, then $f$ maps $[k]$ onto $I$ a.e. For odd $q$, there is a similar list of excluded sequences (cf. [Ro] and Figure 2), and again if $k>1$, then $f$ maps $[k]$ onto $I$ a.e.

In this section, we prove the following theorem.

Theorem 5.1. The process $(I, f, \rho, \mathcal{P})$ is weak Bernoulli.

Set

$$
\begin{array}{r}
\beta_{n}=\sup _{L \geq 1}\left\{\sum_{i, j}\left|\rho\left(A_{i} \cap T^{-n-L}\left(A_{j}\right)\right)-\rho\left(A_{i}\right) \rho\left(A_{j}\right)\right|\right. \\
\left.:\left\{A_{i}\right\} \text { is the set of cylinders of length } L\right\} .
\end{array}
$$

Recall that the process is weak Bernoulli if and only if $\beta_{n} \rightarrow 0$. It is a theorem of H. Berbee [Ber2] that the process is weak Bernoulli if and only if $(I, f, \rho)$ is weakly mixing and there is an $n$ such that $\beta_{n}<1$. See also [Ber1]. In what follows, this is exactly what we show. 
5.1. An induced weak Bernoulli process. Our first step is to show that a certain induced process is weak Bernoulli.

Let $Y=\bigcup_{n=2}^{\infty} P_{n}=(0,2 /(3 \lambda)]$ and define the induced transformation $f_{Y}$ : $Y \rightarrow Y$ by $f_{Y}(y)=f^{n}(y)$, where $n=\inf \left\{k>0: f^{k}(y) \in Y\right\}$. Let $\rho_{Y}$ be the normalized probability measure (thus, $\rho_{Y}(A)=\rho(A) / \rho(Y)$ for $A \subset Y$ ). The dynamical system $\left(Y, f_{Y}, \rho_{Y}\right)$ has a natural generating partition $\mathcal{Q}$, given by

$\left\{Q_{\alpha}: \alpha=\left(a_{1}, \ldots, a_{m}\right) ; a_{1} \geq 2 ; a_{i}<2, i>1 ; \alpha\right.$ contains no forbidden substrings $\}$,

where $Q_{\alpha}=\left[a_{1}, a_{2}, \ldots, a_{m}\right] \cap f^{-m}(Y)$.

Proposition 5.1. The process $\left(Y, f_{Y}, \rho_{Y}, \mathcal{Q}\right)$ is weak Bernoulli.

Proof. By a theorem of R. Adler [A; Theorem, p. 112], it suffices to verify the following conditions:

(i) $f_{Y}$ maps each $Q_{\alpha}$ onto $Y$;

(ii) $f_{Y}$ restricted to each $Q_{\alpha}$ is twice differentiable;

(iii) $\inf _{x \in Y}\left|f_{Y}^{\prime}(x)\right|>1$;

(iv) $\sup _{\alpha} \sup _{x, y \in Q_{\alpha}} \frac{\left|f_{Y}^{\prime \prime}(x)\right|}{\left[f_{Y}^{\prime}(y)\right]^{2}}<\infty$.

All of these conditions are clear with the exception of (iv). E.g., (iii) at once follows from the chain rule, the facts that $\left|f^{\prime}(x)\right|>1$ and that $f_{Y}$ is the $m$-fold composition of $f$ on $Q_{\alpha}$ for $\alpha=\left(a_{1}, \ldots, a_{m}\right)$.

A proof of (iv) can be obtained as follows. Restricting $f_{Y}$ to $Q_{\alpha}=\left[a_{1}, \ldots, a_{m}\right]$, we find that

$$
f_{Y}(x)=f^{m}(x)=\frac{q_{m} x-p_{m}}{-q_{m-1} x+p_{m-1}} .
$$

Notice that $p_{m-1}, p_{m}, q_{m-1}$ and $q_{m}$ are common to all $x \in Q_{\alpha}$.

Thus, $\left|f_{Y}^{\prime}(x)\right|=q_{m-1}^{2} / \Theta_{m-1}^{2}(x)$, where the $\Theta_{n}(x)$ are defined as in Section 4. In fact, another proof of (iii) now follows from this and Theorem 4.2.

To prove (iv), we must bound

$$
\frac{2 q_{m-1}\left(q_{m-1} y-p_{m-1}\right)^{4}}{\left|q_{m-1} x-p_{m-1}\right|^{3}} .
$$

But, this is

$$
2 \Theta_{m-1}(y) \cdot\left[\Theta_{m-1}(y) / \Theta_{m-1}(x)\right]^{3} ;
$$

thus it suffices to bound $\Theta_{m-1}(y) / \Theta_{m-1}(x)$.

Recall that $\psi\left(f^{m-1}(x), q_{m-2} / q_{m-1}\right)=\left(\Theta_{m-2}(x), \Theta_{m-1}(x)\right)$. Hence,

$$
\Theta_{m-1}(y) / \Theta_{m-1}(x)=\frac{q_{m-1}+q_{m-2} f^{m-1}(x)}{q_{m-1}+q_{m-2} f^{m-1}(y)} \cdot \frac{\left|f^{m-1}(y)\right|}{\left|f^{m-1}(x)\right|} .
$$

Notice that for $x, y \in Q_{\alpha}$, one has that $r_{m}(x)=\left|a_{m}\right|=r_{m}(y)$, with either $\varepsilon_{m}(x)=$ $\varepsilon_{m}(y)=-1$ or $\varepsilon_{m}(x)=\varepsilon_{m}(y)=+1$ and $a_{m}=1 ; r_{m+1}(x) \geq 2 ; r_{m+1}(y) \geq 2$; and $\varepsilon_{m+1}(x)=\varepsilon_{m+1}(y)=+1$. If $\varepsilon_{m}(x)=\varepsilon_{m}(y)=-1$ we find that

$$
\Theta_{m-1}(y) / \Theta_{m-1}(x) \leq \frac{q_{m-1}-q_{m-2} /\left(r_{m} \lambda+2 /(3 \lambda)\right)}{q_{m-1}-q_{m-2} /\left(r_{m} \lambda\right)} \cdot \frac{r_{m} \lambda+2 /(3 \lambda)}{r_{m} \lambda} .
$$

The first of these factors is easily seen to be less than 2 ; the second factor is also easily bounded. If $\varepsilon_{m}(x)=\varepsilon_{m}(y)=+1$, then each of $f^{m-1}(x)$ and $f^{m-1}(y)$ is between $2 /(3 \lambda)$ and $1 / \lambda$; a bound follows easily. 
Now, the process $(\Omega, T, \nu)$ is a tower over the weak Bernoulli (and a fortiori ergodic) $\left(Y, T_{Y}, \nu_{Y}\right)$, and therefore we have the following.

Corollary 5.1. The process $(\Omega, T, \nu)$ is ergodic.

5.2. A small coupling constant $\beta_{n}$. Now we pass to the natural extension $(\Omega, T, \nu)$. The partition $\mathcal{P}$ and the subset $Y$ have their corresponding meanings in this context. The induced dynamical system $\left(Y, T_{Y}, \nu_{Y}\right)$ has a natural partition $\mathcal{Q}=\left\{Q_{\alpha}\right\}$. Notice that each partition element $Q_{\alpha}$ corresponds to a cylinder $[\alpha]$ in $(\Omega, T, \nu)$.

There is an interpretation of $(\Omega, T, \nu, \mathcal{P})$ as the distribution of a stochastic process; see [B] for more details. A sequence of random variables $\left\{X_{n}: n \in \mathbf{Z}\right\}$ has distribution $(\Omega, T, \nu, \mathcal{P})$ if $X_{n} \in \mathbf{Z} \backslash\{0\}$ for all $n \in \mathbf{Z}$ and if $\operatorname{Prob}\left[X_{n}=a_{0}, X_{n+1}=\right.$ $\left.a_{1}, \ldots, X_{n+m}=a_{m}\right]=\nu\left(\left[a_{0}, \ldots, a_{m}\right]\right)$ for each $n \in \mathbf{Z}, m \geq 0, a_{i} \in \mathbf{Z} \backslash\{0\}$.

It is a result of Berbee that the process is weak Bernoulli if and only if there is a sequence of joint random variables (called a coupling) $\left\{\left(X_{n}, X_{n}^{\prime}\right)\right\}$ such that

(1) the distribution of both $\left\{X_{n}\right\}$ and of $\left\{X_{n}^{\prime}\right\}$ is $(\Omega, T, \nu, \mathcal{P})$;

(2) $\left\{X_{n}^{\prime}: n \leq 0\right\}$ is stochastically independent of $\left\{X_{n}: n \in \mathbf{Z}\right\}$;

(3) $\operatorname{Prob}\left[X_{n}=X_{n}^{\prime}\right.$ for all but finitely many positive $\left.n\right]=1$.

In fact Berbee [Ber1] showed more. For any process, weak Bernoulli or not, we can find a coupling $\left\{\left(X_{n}, X_{n}^{\prime}\right)\right\}$ satisfying (1) and (2) above as well as

$$
\operatorname{Prob}\left[X_{m}=X_{m}^{\prime}, \forall m \geq n\right]=\beta_{n} .
$$

In particular, $\beta_{m}<1$ for some $m$ if and only if we can find a coupling $\left\{\left(X_{n}, X_{n}^{\prime}\right)\right\}$ satisfying (1) and (2) as well as

$\left(3^{\prime}\right) \operatorname{Prob}\left[X_{n}=X_{n}^{\prime}\right.$ for all but finitely many positive $\left.n\right]>0$.

Since the dynamical system $\left(Y, T_{Y}, \nu_{Y}, \mathcal{Q}\right)$ is weak Bernoulli, there is a coupling as above. Note that the distribution of this coupling gives a probability measure, say $\xi_{Y}$, on $Y \times Y$ for which both marginal measures are $\nu_{Y}$. If $A, B \subset Y$, define $\xi(A \times B)=\nu(Y)^{2} \xi_{Y}(A \times B)$. If either $A \subset \Omega \backslash Y$ or $B \subset \Omega \backslash Y$, define $\xi(A \times B)=$ $\nu(A) \nu(B)$. This determines $\xi$ and gives a distribution for $\left\{\left(X_{n}, X_{n}^{\prime}\right): n \in \mathbf{Z}\right\}$. This coupling satisfies (1) and (2). From our construction, we can group symbols in the coupling $\left\{\left(X_{n}, X_{n}^{\prime}\right)\right\}$ so as to obtain this previous coupling for $Y$. Thus, the coupling also satisfies

$\left(3^{\prime \prime}\right) \operatorname{Prob}\left[\exists N \in \mathbf{Z}\right.$ such that $X_{n}=X_{n+N}^{\prime}$ for all but finitely many $\left.n>0\right]>0$.

There is a (random) $N$ such that for almost every sequence $\left\{\left(X_{n}, X_{n}^{\prime}\right), n \in \mathbf{Z}\right\}$, with $X_{0} \geq 2$ and $X_{0}^{\prime} \geq 2$, one has $\#\left\{n>0: X_{n} \neq X_{n+N}^{\prime}\right\}<\infty$. One would expect $N \neq 0$ since the coupling for $\left(Y, T_{Y}, \nu_{Y}, \mathcal{Q}\right)$ matches various $Q_{\alpha}$, but two such $Q_{\alpha}$ might correspond to cylinders $[\alpha]$ of different lengths.

Fix an $N$ so that $\operatorname{Prob}\left[\#\left\{n>0: X_{n} \neq X_{n+N}^{\prime}\right\}<\infty\right]>0$. Now let $X_{n}^{\prime \prime}=$ $X_{n+N}$. Then $\left\{\left(X_{n}^{\prime \prime}, X_{n}^{\prime}\right)\right\}$ is a coupling satisfying $(1),(2)$ and $\left(3^{\prime}\right)$. Hence, there is some $n$ such that $\beta_{n}<1$.

5.3. Weakly mixing. To complete the proof of the theorem, we need only show that $(\Omega, T, \nu)$ is weakly mixing.

Define $\mathcal{S}: \Omega \rightarrow[2]$ by

$$
\mathcal{S}(x)_{i}= \begin{cases}x_{i-1}, & i>0 \\ 2, & i=0, \\ x_{i}, & i<0 .\end{cases}
$$


Thus, $\mathcal{S}$ inserts a 2 into the symbolic representation by $\mathcal{P}$ of $x \in \Omega$.

Lemma 5.1. There exist constants $c$ and $d$, both strictly between 1 and $\infty$, such that

(A) If $\left[a_{0}, \ldots, a_{m}, b_{0}, \ldots, b_{m}\right]$ is a cylinder set of positive $\nu$-measure, then

$$
1 / c \leq \frac{\nu\left(\left[a_{1}, \ldots, a_{m}, b_{1}, \ldots, b_{m}\right]\right)}{\nu\left(\left[a_{1}, \ldots, a_{m}\right]\right) \cdot \nu\left(\left[b_{1}, \ldots, b_{m}\right]\right)} \leq c
$$

(B) If $A \subset[2]$ is measurable, then

$$
1 / d \leq \frac{\nu\left(\mathcal{S}^{-1}(A)\right)}{\nu(A)} \leq d .
$$

Proof. The invariant density $h(x, y)=C /(1+x y)^{2}$ on $\Omega$ has projections

$$
h_{x}(t)=\int h(t, y) d y \quad \text { and } \quad h_{y}(t)=\int h(x, t) d x .
$$

These are the invariant densities on $[-\lambda / 2, \lambda / 2]$ and $[0, R]$, respectively. Each of $h, h_{x}$ and $h_{y}$ is bounded away from 0 and $\infty$.

Let $A=\left[a_{1}, \ldots, a_{m}\right], B=\left[b_{1}, \ldots, b_{n}\right]$ and $D=\left[a_{1}, \ldots, a_{m}, b_{1}, \ldots, b_{n}\right]$. Thus,

$$
\begin{aligned}
\frac{\nu(D)}{\nu(A) \nu(B)} & =\frac{\iint_{T^{m}(D)} h(x, y) d x d y}{\int_{T^{m}(A)} h_{y}(t) d t \cdot \int_{B} h_{x}(t) d t} \\
& \leq \frac{\sup \{h(x, y)\}}{\inf \left\{h_{y}(t)\right\} \cdot \inf \left\{h_{x}(u)\right\}} .
\end{aligned}
$$

The uniform bound from below is similarly demonstrated. Thus, (A) follows.

To prove (B), it suffices to consider cylinder sets. But,

$$
\begin{aligned}
& \frac{\nu\left(\left[a_{1}, \ldots, a_{m}, b_{1}, \ldots, b_{n}\right]\right)}{\nu\left(\left[a_{1}, \ldots, a_{m}, 2, b_{1}, \ldots, b_{n}\right]\right)} \\
& =\frac{\nu\left(\left[a_{1}, \ldots, a_{m}, b_{1}, \ldots, b_{n}\right]\right)}{\nu\left(\left[a_{1}, \ldots, a_{m}\right]\right) \cdot \nu\left(\left[b_{1}, \ldots, b_{n}\right]\right)} \cdot \frac{\nu\left(\left[a_{1}, \ldots, a_{m}\right]\right) \cdot \nu\left(\left[b_{1}, \ldots, b_{n}\right]\right) \cdot \nu([2])}{\nu\left(\left[a_{1}, \ldots, a_{m}\right]\right) \cdot \nu\left(\left[2, b_{1}, \ldots, b_{n}\right]\right)} \\
& \quad \cdot \frac{\nu\left(\left[a_{1}, \ldots, a_{m}\right]\right) \cdot \nu\left(\left[2, b_{1}, \ldots, b_{n}\right]\right)}{\nu\left(\left[a_{1}, \ldots, a_{m}, 2, b_{1}, \ldots, b_{n}\right]\right) \cdot \nu([2])} .
\end{aligned}
$$

Thus, $d$ may be found using part (A) of this lemma.

Lemma 5.2. The system $(T, \Omega, \nu)$ is weakly mixing.

Proof. It suffices to show that there is no rotation in the system, i.e., that for the action of $T$ on $f \in L^{\infty}(\nu)$ given by $[T f](x)=f(T x)$, there are no eigenvalues on $S^{1} \subset \mathbf{C}$.

Let $f \in L^{\infty}(\nu)$ and $\xi \in S^{1}$ be such that $T f=\xi f$. Let $\tilde{f}(x)=f(\mathcal{S} x)$. The function $\tilde{f} \in L^{\infty}(\nu)$ is well defined, as the previous lemma guarantees that if $f=g$ then $\tilde{f}=\tilde{g}$ a.e. To see this, note that if $E=\{x \mid f(x) \neq g(x)\}$, then $\tilde{f}=\tilde{g}$ off of $\mathcal{S}^{-1}(E)$. The previous lemma implies that $\nu\left(S^{-1}(E)\right)=0$, as $\nu(E)=0$.

Since $\xi \in S^{1}$, we can find $n_{i} \uparrow \infty$ such that $\xi^{n_{i}} \rightarrow 1$. But then, $T^{n_{i}} f=\xi^{n_{i}} f \rightarrow f$ and $T^{-n_{i}} f=\xi^{-n_{i}} f \rightarrow f$, as well as $T^{n_{i}} \tilde{f} \rightarrow \tilde{f}$ and $T^{-n_{i}} \tilde{f} \rightarrow \tilde{f}$. Due to the action of $\mathcal{S}$, we also have $T^{n_{i}} \tilde{f} \rightarrow T f$ (measure) and $T^{-n_{i}} \tilde{f} \rightarrow f$ (measure). But, this implies that $T f=\tilde{f}=f$ a.e., hence that $f$ is constant, as we already know that the system is ergodic. Therefore, the system $(T, \Omega, \nu)$ is weakly mixing. 


\section{REFERENCES}

[A] R. Adler, Continued fractions and Bernoulli trials, in Ergodic Theory (A Seminar), J. Moser, E. Phillips and S. Varadhan, eds., Courant Inst. of Math. Sci. (Lect. Notes 110), 1975, New York. MR 58:6177

[B] A. Beardon, The Geometry of Discrete Groups, Graduate Texts in Mathematics 91, Springer-Verlag 1983, New York. MR 85d:22026

[Ber1] H.C.P. Berbee, Random walks with stationary increments and renewal theory, Math. Centre Tracts 112, Mathematisch Centrum, Amsterdam 1979. MR 81e:60093

[Ber2] H.C.P. Berbee, Periodicity and absolute regularity, Israel J. Math. 55 (1986), 289-304. MR 88b:60088

[GH] K. Gröchenig and A. Haas, Backward continued fractions and their invariant measures, Canad. Math. Bull. 39 (1996), 186-198. MR 97h:11077

[HS] A. Haas and C. Series, Hurwitz constants and Diophantine approximation on Hecke groups, J. London Math. Soc. 34 (1986), 219-234. MR 87m:11060

[JK] H. Jager and C. Kraaikamp, On the approximation by continued fractions, Indag. Math. 51 (1989), 289-307. MR 90k:11084

[K1] C. Kraaikamp, The distribution of some sequences connected with the nearest integer continued fraction, Indag. Math. 49 (1987), 177-191. MR 88j:11045

[K2] C. Kraaikamp, A new class of continued fraction expansions, Acta Arith. 57 (1991), 1-39. MR 92a: 11090

[KL] C. Kraaikamp and A. Lopes, The theta group and the continued fraction with even partial quotients, Geom. Dedicata 59 (1996), 293-333. MR 97g:58135

[L1] J. Lehner, Diophantine approximation on Hecke groups, Glasgow Math. J. 27 (1985), 117-127. MR 87e:11079

[L2] J. Lehner, The local Hurwitz constant and Diophantine approximation on Hecke groups, Math. Comp. 55 (1990), 765-781. MR 91c:11036

[N1] H. Nakada, Metrical theory for a class of continued fraction transformations, Tokyo J. Math. 4 (1981), 399-426. MR 83k:10095

[N2] H. Nakada, Continued fractions, geodesic flows and Ford circles, in: Algorithms, Fractals and Dynamics, Ed. Y. Takahashi, Plenum Press, 1995, 179-191. MR 97g:11086

[NIT] H. Nakada, Sh. Ito, S. Tanaka, On the invariant measure for the transformations associated with some real continued-fractions, Keio Engrg. Rep. 30 (1977), 159-175. MR 58: 16574

[R] G. J. Rieger, Mischung und Ergodizität bei Kettenbrüchen nach nächsten Ganzen, J. Reine Angew. Math. 310 (1979), 171-181. MR 81c:10066

[Ro] D. Rosen, A class of continued fractions associated with certain properly discontinuous groups, Duke Math. J. 21 (1954), 549-563. MR 16:458d

[S] T. A. Schmidt, Remarks on the Rosen $\lambda$-continued fractions, in Number theory with an emphasis on the Markoff spectrum, A. Pollington, W. Moran, eds., Dekker, New York, 1993, 227-238. MR 94i:11034

[W] H. Weber, Lehrbuch der Algebra, I, 2nd edition, Viehweg, Braunschweig, 1898.

[Wo] J. Wolfart, Diskrete Deformation Fuchsscher Gruppen und ihrer automorphen Formen, J. Reine Angew. Math. 348 (1984), 203-220. MR 85c:11044

Department of Mathematics, Oregon State University, Corvallis, Oregon 97331

E-mail address: burton@math.orst.edu

Technische Universiteit Delft \& Thomas Stieltues Institute of Mathematics, ITS (SSOR), Mekelweg 4, 2628 CD Delft, the Netherlands

E-mail address: c.kraaikamp@its.tudelft.nl

Department of Mathematics, Oregon State University, Corvallis, Oregon 97331

E-mail address: toms@math.orst.edu 